\title{
Dynamique de l'immunité passive et stratégie de vaccination des veaux contre la peste bovine*
}

\author{
V.N. Tanya ${ }^{1}$ A. Keng ${ }^{1}$ O. Messine \\ A. Ngangnou ${ }^{2}$ N. Zoyem ${ }^{2}$ I.N.D. Kulu ${ }^{1}$
}

Mots-clés

Bovin - Veau - Peste bovine - Vaccination - Immunité passive - Immunologie - Neutralisation - Anticorps Cameroun.

\section{Résumé}

Afin de déterminer la dynamique de l'immunité passive contre la peste bovine, des veaux nés de mères immunisées ont été suivis sérologiquement de la naissance à l'âge d'un an. L'interférence éventuelle de ces anticorps avec la vaccination a été évaluée par le biais d'une vaccination des veaux présentant un large éventail de titres d'anticorps. Les anticorps d'origine maternelle ont diminué de manière progressive avec l'âge. A 8 mois, seuls 7 p. 100 des veaux en possédaient encore. Après cet âge, ces anticorps n'étaient plus détectables. L'analyse par régression du titre des anticorps maternels avec l'âge des veaux a montré une demi-vie de 48,01 jours. Ces anticorps ont empêché le développement d'une réponse à la vaccination, laquelle était inversement proportionnelle au niveau d'anticorps maternels présents au moment de la vaccination. II a aussi été observé que les veaux ayant un titre élevé d'anticorps maternels n'avaient non seulement pas répondu à la vaccination, mais leur titre sérologique avait aussi décru. Ces résultats sont importants pour le planning des campagnes d'éradication de la peste bovine.

\section{INTRODUCTION}

La peste bovine est une maladie dévastatrice des bovins avec de graves conséquences sociales et économiques. C'est un vieux fléau des bovidés (bovins et buffles). C'est une maladie aiguë, fébrile, très contagieuse, caractérisée par un état typhique plus ou moins accusé, une inflammation des muqueuses, celle du tractus digestif en particulier, accompagnée d'érosion et de nécrose (8). La maladie se caractérise par une courte fièvre, une lymphocytolyse, une stomatite érosive et des diarrhées. Dans les populations bovines hautement sensibles, tous les animaux infectés tombent malades et la mortalité peut atteindre 90 p. 100 (6). Bien qu'étant éradiquée dans beaucoup de régions du monde, elle reste une des maladies économiquement importantes en Afrique et en Asie (15).

* Etudes financées par l'Association des Universités Partiellement ou Entièrement de Langue Française, Université des Réseaux d'Expression Française (AUPELFUREF) dans le cadre de l'action « Laboratoires Associés Francophones (LAF) » du Fonds Francophone de la Recherche

1. Laboratoire Associé Francophone (LAF 309), Centre Régional de Recherche Agricole pour le Développement, Wakwa, BP 65, Ngaoundéré, Cameroun

Fax : (237) 251557

2. Laboratoire National Vétérinaire (LANAVET), Boklé-Garoua, BP 503, Garoua, Cameroun
Malgré de nombreux efforts visant à son contrôle par la vaccination dans les années 1960 et 1976, elle a resurgi dans la majeure partie de l'Afrique au nord de l'Equateur à la fin des années 1970 et au début des années $1980(9,13)$. Cette résurgence serait due à l'existence d'une mauvaise couverture vaccinale dans de nombreuses zones. Il est aussi possible que la vaccination des veaux n'ait pas été complètement effective, résultant en une large population bovine susceptible. Il n'existe pas ou peu d'informations sur la dynamique de l'immunité passive au virus de la peste bovine chez les veaux et l'effet de cette immunité passive sur leur vaccination précoce. Cette information est primordiale pour déterminer l'âge optimum pour la vaccination contre la peste bovine. L'objet de cette étude est de fournir cette information par le suivi de la naissance à un an d'âge des veaux nés de vaches immunisées. Afin de démontrer l'effet des anticorps maternels sur la réponse immunitaire post-vaccinale, des veaux possédant divers niveaux d'anticorps maternels ont aussi été vaccinés.

\section{MATERIEL ET METHODES}

\section{Veaux expérimentaux}

Tous les veaux de l'expérience étaient nés dans un laps de temps assez court grâce à l'insémination artificielle avec de la semence fraîche de taureaux de race Goudali. L'objectif était de minimiser 
les effets et les difficultés pouvant découler des différences de saison de naissance. Pour ce faire, les vaches Goudali ont été sélectionnées sur la base de leurs performances de reproduction, leur cyclicité et leur non gravidité dans les troupeaux du Centre régional de la recherche agricole pour le développement de Wakwa, Cameroun. Elles ont ensuite été synchronisées avec des progestagènes (implants sous-cutanés Synchro Mate $\mathrm{B}^{\mathrm{ND}}$ ) et/ou de la prostaglandine $F 2 \alpha$ (ou analogue). Neuf jours après le début du traitement, 330 UI de PMSG ont été injectées par voie intramusculaire. L'insémination artificielle a été réalisée à l'œstrus, détecté dans les 96 h qui ont suivi l'arrêt du traitement. Le diagnostic de gestation par toucher rectal a eu lieu à 90 et à 120 jours post-insémination.

\section{Protocole expérimental}

Les veaux obtenus ont été utilisés dans les expériences suivantes :

\section{Etude de la dynamique de l'immunité passive} contre la peste bovine chez les veaux

Des échantillons de sang ont été collectés par ponction jugulaire de chaque veau 24-36 $\mathrm{h}$ après la naissance et une fois toutes les deux semaines jusqu'à l'âge de 12 mois. Les sérums obtenus ont été conservés à $-20{ }^{\circ} \mathrm{C}$ jusqu'au titrage des anticorps antibovipestiques. Des données obtenues ont été analysées par régression linéaire, et la demi-vie des anticorps colostraux anti-peste bovine déterminée en utilisant la méthode décrite par Bryan et coll. (4).

\section{Effet des anticorps maternels}

\section{sur la réponse immunitaire post-vaccinale}

Afin de mener à bien cette étude, des veaux Goudali nés en station (mais différents de ceux utilisés dans l'étude dynamique) ont été regroupés en quatre classes d'âge (tableau I). Ils ont été vaccinés contre la peste bovine et des échantillons de sérum ont été prélevés au moment de la vaccination et deux semaines plus tard. Les sérums obtenus ont été conservés à $-20^{\circ} \mathrm{C}$ jusqu'au titrage des anticorps. Les titres d'anticorps ont été déterminés et les données analysées (régression et analyse de variance) afin d'évaluer les effets des anticorps maternels sur la vaccination.

\section{Vaccination}

Toutes les vaches gravides ont été vaccinées entre le sixième et le huitième mois contre la peste bovine. Le vaccin bovipestique utilisé a été produit au Laboratoire national vétérinaire (LANAVET), Boklé-Garoua, Cameroun. C'est un vaccin thermostable produit à partir de cellules Vero (7). Le contrôle de la réponse post-vaccinale chez les mères vaccinées a été effectué avant le début des mises bas.

\section{Alimentation}

Les vaches et leurs veaux ont été maintenus sur pâturage de savane naturelle en saison des pluies, sur foin de savane naturelle et complément azoté à base de tourteau de coton en saison sèche.

\section{Titrage des anticorps au virus de la peste bovine}

Les sérums ont été testés par la méthode de séroneutralisation basée sur un titre constant de virus et un titre de sérum variable dans les plaques microwell stériles à fond plat à 96 cupules (11, 12). Le virus bovipestique, souche RPKO, $99^{\mathrm{e}}$ passage, a été préalablement titré dans un volume de $50 \mu \mathrm{l}$ sur cellules Vero. Le titrage a permis la détermination de la dilution du virus correspondant à $100 \mathrm{DICT}_{50}$ dans un volume de $50 \mu \mathrm{l}$. Les cellules ont été cultivées en milieu MEM Glasgow, additionné de 10 p. 100 de sérum de veau, 2 p. 100 du mélange pénicilline/streptomycine (pénicilline G $10000 \mathrm{UI} / \mathrm{ml}$, streptomycine $10000 \mathrm{mg} / \mathrm{ml}$ ), 1 p. $100 \mathrm{de}$ sulfate de gentamicine $(50 \mathrm{mg} / \mathrm{ml}), 2$ p. 100 amphotéricine B $(250 \mu \mathrm{g} / \mathrm{ml})$ et ajusté à $\mathrm{pH} 7,2$.

Les sérums à examiner ont été décomplémentés, testés en double à partir de la dilution $1 / 10^{\mathrm{e}}$, au contact de $50 \mu \mathrm{l}$ de virus bovipestique à la dilution donnant $100 \mathrm{DICT}_{50}$. Le mélange ainsi obtenu a été incubé dans une étuve à $\mathrm{CO}_{2}$ à $37^{\circ} \mathrm{C}$ pendant une heure. Après contact, chaque cupule a reçu $100 \mu \mathrm{l}$ de milieu contenant environ 25000 cellules Vero. L'incubation à l'étuve à $\mathrm{CO}_{2}$ a été poursuivie pendant six jours pour obtenir le titre de chaque sérum. Les titres obtenus ont été exprimés comme le logarithme de l'inverse de la dilution de sérum ayant produit 50 p. 100 de protection $\left(\mathrm{VN}_{50}\right)$ à une dose de $100 \mathrm{DICT}_{50}$ de virus bovipestique. Tous les tests ont été conduits en double et la valeur moyenne des dilutions calculée.

\section{RESU LTATS}

\section{Etude de la dynamique de l'immunité passive contre la peste bovine chez les veaux}

Quarante-quatre veaux ont été utilisés pour cette étude. Les mères des veaux avaient au préalable été vaccinées 2 à 4 mois avant le vêlage. Malgré la grande dispersion dans l'intervalle entre la vaccination des mères et la naissance des veaux, 42 des 44 veaux $(95,45$ p. 100) âgés de 24 à 36 h possédaient des anticorps d'origine maternelle au virus de la peste bovine, de l'ordre de 1,6 à $3,12 \log _{10} \mathrm{VN}_{50}$. Les deux veaux restants ne présentaient pas d'anticorps détectables. Il est probable que ces veaux n'aient pas pris

\section{Tableau I}

Effet des anticorps maternels sur la réponse immunitaire post-vaccinale

$\begin{array}{ccccc}\text { Groupe d'animaux } & \text { Age (jours) } & \text { Nb. de veaux } & \begin{array}{c}\text { Titre des anticorps } \\ \text { maternels* } \\ \text { (moyenne } \pm \text { e.t.) }\end{array} & \begin{array}{c}\text { Réponse } \\ \text { post-vaccinale** } \\ \text { (moyenne } \pm \text { e.t.) }\end{array} \\ \text { I } & 0-90 & 29 & 2,05 \pm 0,66 & -0,17 \pm 0,65 \\ \text { II } & 91-180 & 25 & 1,52 \pm 0,88 & 0,13 \pm 1,30 \\ \text { III } & 181-270 & 20 & 0,98 \pm 1,40 & 1,19 \pm 1,67 \\ \text { IV } & >270 & & 0 & 2,58 \pm 0,62\end{array}$


de colostrum. La figure 1 présente la courbe de la dynamique des anticorps colostraux en fonction de l'âge. Le titre des anticorps maternels des veaux montre une diminution progressive avec l'âge. A 126 jours d'âge, seuls 52 p. 100 des veaux possédaient encore des anticorps maternels. Cette proportion a continué à décroître (38 p. 100 à 140 jours) de telle sorte qu'à l'âge de 238 jours (environ 8 mois), seuls 7 p. 100 des veaux ont encore montré la présence d'anticorps (titre moyen de $1,01 \log _{10} \mathrm{VN}_{50}$ ) d'ailleurs difficilement détectables. Après cet âge, aucun veau n'a plus présenté d'anticorps maternels détectables. L'analyse par régression des anticorps maternels avec l'âge du veau montre une demi-vie de 48,01 jours. La régression linéaire, quant à elle, a un ajustement significatif $(\mathrm{p}<0,001)$.

\section{Réponse des veaux à une primo-vaccination contre la peste bovine en présence d'anticorps maternels}

Les veaux utilisés dans cette étude présentaient une grande dispersion dans le titre des anticorps maternels $\left(0-3,12 \log _{10} \mathrm{VN}_{50}\right)$ au moment de la vaccination. La réponse (augmentation ou diminution post-vaccinale du titre des anticorps) à la vaccination a été inversement proportionnelle au niveau des anticorps maternels présents au moment de la vaccination (tableau I, figure 2). La vaccination des veaux en présence d'anticorps d'origine maternelle produisait une différence significative $(\mathrm{p}<0,001)$ dans la réponse parmi les quatre groupes de veaux. Les veaux du premier groupe, âgés de 0 à 90 jours et possédant un haut niveau d'anticorps ma- ternels, n'ont pas répondu à la vaccination et, de plus, ont présenté une diminution dans le titre de sérum (tableau I). Les veaux des groupes II et III, âgés respectivement de 91 à 180 et 181 à 270 jours, qui présentaient un niveau d'anticorps d'origine maternelle relativement bas ont répondu à la vaccination avec des augmentations du titre inversement proportionnelles au niveau des anticorps maternels au moment de la vaccination. Une réponse excellente à la première vaccination contre le virus de la peste bovine a été obtenue avec les veaux âgés de plus de 270 jours (environ 9 mois) qui ne présentaient pas d'anticorps d'origine maternelle.

L'analyse par régression (figure 2) de l'augmentation ou de la diminution post-vaccinale du titre des anticorps individuels par rapport au niveau des anticorps maternels présents au moment de la vaccination a montré un ajustement significatif $(\mathrm{p}<0,001)$.

\section{DISCUSSION ET CONCLUSION}

Les différentes études ci-dessus ont montré que les anticorps maternels contre le virus de la peste bovine diminuaient progressivement avec l'âge. La réponse à la vaccination a été inversement proportionnelle au niveau des anticorps maternels présents au moment de la vaccination. Des titres élevés d'anticorps maternels interdisent la prise du vaccin. Le vaccin vivant antibovipestique connu pour son efficacité doit cependant être administré selon un protocole bien établi. Le contrôle de la peste bovine pourrait dès lors être amélioré si le moment optimum de son injection était respecté.

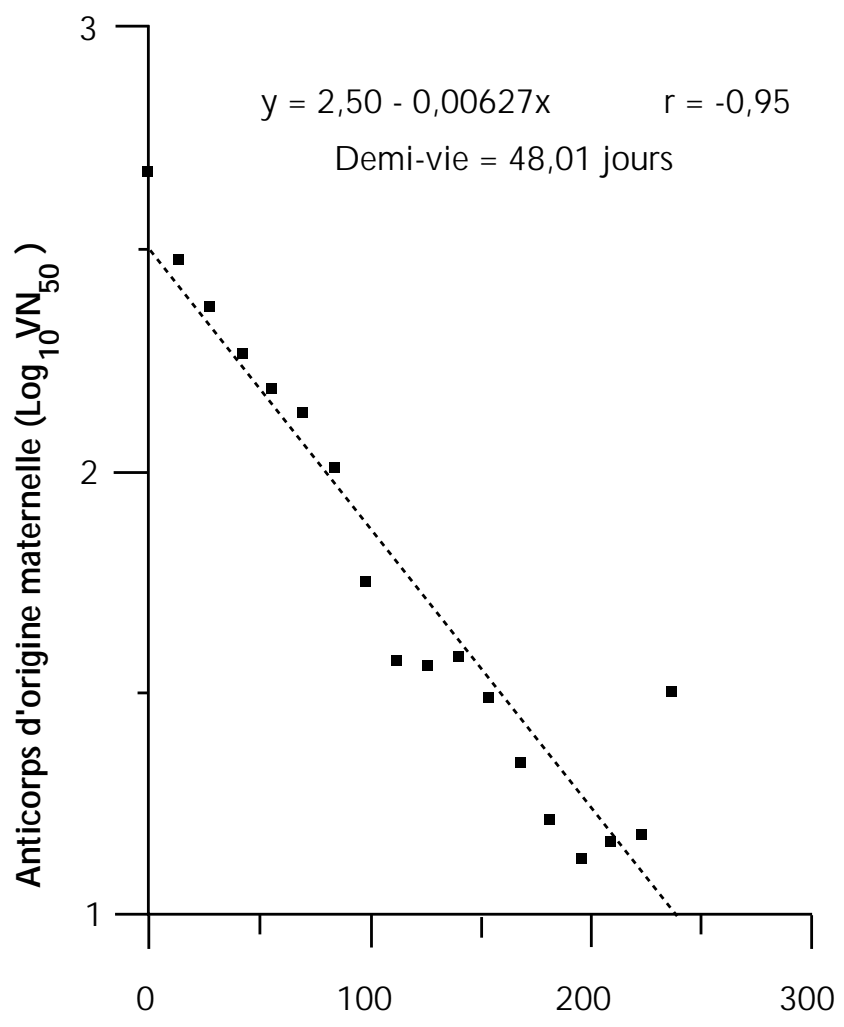

Age du veau (jours)

Figure 1 : déclin des anticorps d'origine maternelle avec l'âge du veau.

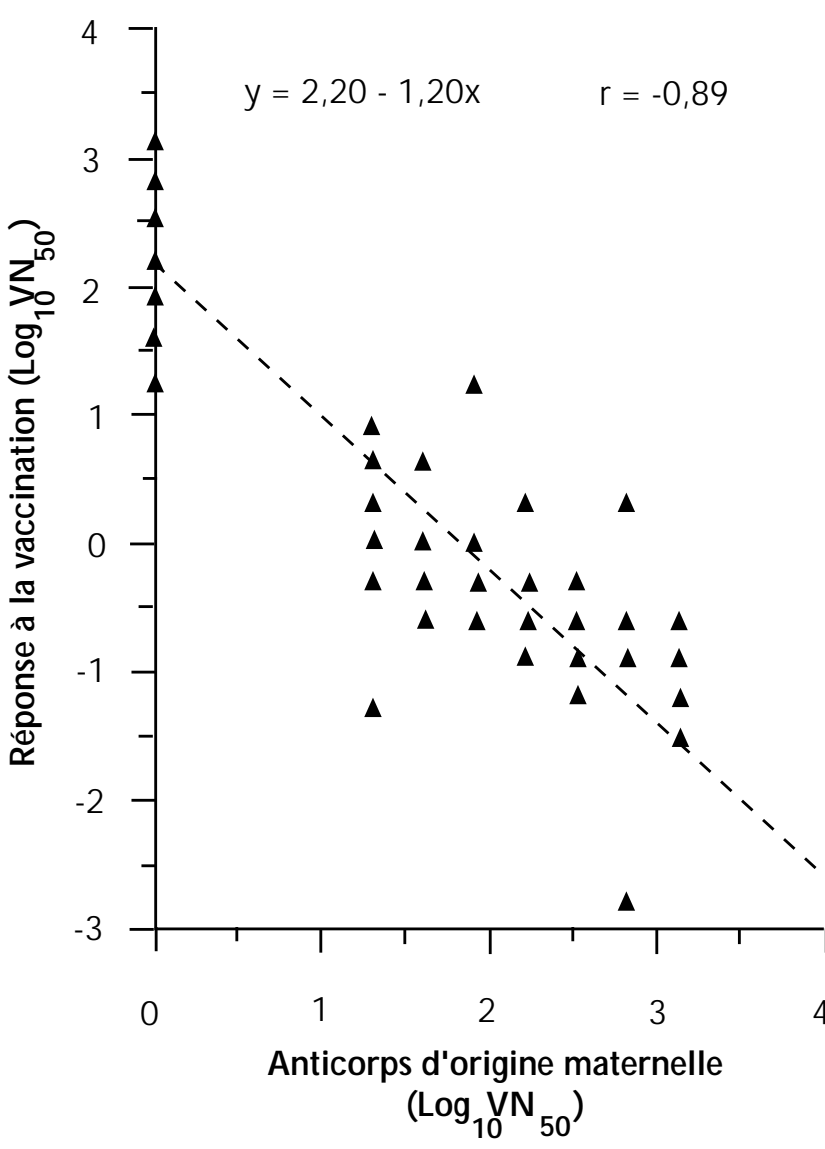

Figure 2 : réponse à la primo-vaccination en présence d'anticorps d'origine maternelle. 
Ces résultats, comme ceux de Couacy-Hymann (5) et Strickland (16), sont indicatifs du fait que l'immunité passive chez la plupart des veaux persiste pendant une période inférieure à six mois. Ils diffèrent toutefois de ceux de Brown $(2,3)$ qui donnent un intervalle plus large, de 4 à 8 mois. Dans la présente étude, à 126 jours (4,2 mois), 52 p. 100 des veaux possédaient encore des anticorps d'origine maternelle. Ce nombre est supérieur aux 28 et 47 p. 100 rapportés par Couacy-Hymann (5) en Côte d'Ivoire sur des veaux âgés de 4 mois et issus de deux troupeaux différents.

Il a été trouvé, dans cette étude, une demi-vie de 48,01 jours alors que Brown $(2,3)$ rapporte une demi-vie de 37 jours. Les différences dans les méthodes d'estimation des indices de neutralisation peuvent être à l'origine des différences entre les résultats de cette étude, où une technique de microtitrage de culture tissulaire a été utilisée, et ceux des études de Brown qui a conduit ses tests de neutralisation sur des lapins.

Ces études ont montré que la vaccination des veaux âgés de 0 à 180 jours (0 à 6 mois) n'est pas totalement effective à cause de la présence d'anticorps d'origine maternelle relativement élevés. Cette situation pourrait conduire à l'établissement d'une large population bovine sensible. Il a été observé que la peste bovine se maintient dans de larges populations bovines où les animaux sensibles sont renouvelés à un rythme élevé. Dans ces populations, la maladie atteint généralement les jeunes animaux ayant perdu leurs anticorps maternels et n'ayant pas encore été vaccinés (14).

Ces études montrent que la peste bovine pourrait aussi être la maladie des animaux ayant été vaccinés précocement, au moment où le niveau des anticorps maternels était encore très élevé. En vue d'éviter cette situation, les veaux ayant été vaccinés avant l'âge de 6 mois devraient recevoir une deuxième vaccination à l'âge d'un an afin de pouvoir être totalement protégés contre la peste bovine.

Des travaux récents de séro-surveillance au Cameroun ont montré que près de 60 à 73 p. 100 des bovins sont protégés contre la peste bovine dans la province de l'Adamaoua, la zone d'élevage bovine par excellence du pays (10). Cette protection est indicative du fait que la plupart des veaux nés dans la province portent des anticorps antibovipestiques d'origine maternelle. Ces veaux pourraient ne pas être complètement protégés contre la peste bovine si la vaccination avait lieu avant l'âge de six mois. Les campagnes annuelles de vaccination telles que celles pratiquées au Cameroun pourraient laisser des milliers de veaux sans protection contre la peste bovine, ce qui pourrait contribuer à la résurgence de la maladie dans le pays.

La recherche sur la peste bovine à la fin des années 80 et au début des années 90 s'est focalisée sur la production de vaccins thermostables $(1,7,17)$ qui ne dépendraient pas de la « chaîne de froid» comme la plupart des vaccins actuels. L'information apportée par les présentes études sera complémentaire à ces efforts.

\section{Remerciements}

Les auteurs sont reconnaissants à l'AUPELF-UREF qui a financé ce travail. Ils remercient Dr Abdoulkadiri Souley, Directeur général du LANAVET, et Dr J.T. Banser, Directeur de l'ex-IRZV, dont l'aide et l'intérêt ont permis de mener à bien ce travail, ainsi que MM. Nguini Atanga et Hamet Moussa pour leur assistance technique.

\section{BIBLIO GRAPHIE}

1. BELSHAM G.J., ANDERSON E.C., MURRAY P.K., ANDERSON J., BARRETT T., 1989. Immune response and protection of cattle and pigs generated by a vaccinia virus recombinant expressing the $F$ protein of rinderpest virus. Vet. Rec., 124: 655-658.

2. BROWN R.D., 1958. Rinderpest immunity in calves. I. The acquisition and persistence of maternally derived antibody. J. Hyg. (Lond.), 56: 427-434.

3. BROWN R.D., 1958. Rinderpest immunity in calves. II. Active immunisation. J. Hyg. (Lond.), 56: 435-444.

4. BRYAN M., ZIMMERMAN J.J., BERRY W.J., 1990. The use of halflives and associated confidence intervals in biological research. Vet. Res. Comm., 14: 235-240.

5. COUACY-HYMANN E., 1994. La lutte contre la peste bovine en Côte d'Ivoire. Coûts et bénéfice des campagnes de prophylaxie. Problèmes posés par son éradication, vol. I et II. Thèse doct. Sci., Université de Paris XII, Paris, France.

6. FENNER F., BACHMANN P.A., GIBBS E.P.J., MURPHY F.A., STUDDERT M.J., WHITE D.O., 1987. Veterinary virology. London, UK, Academic Press, p. 496-503.

7. MARINER J.C., VAN DER ENDE M., HOUSE J.A., MEBUS C.A., SALIFOU S., STEM C., 1990. The serological response to a thermostable Vero cell adapted rinderpest vaccine under field conditions in $\mathrm{Niger}$. Vet. Microbiol., 22: 119-127.

8. MORNET P., 1971. La peste bovine. In : Traité des maladies à virus des animaux, tome III/1. Maisons-Alfort, France, Vigot Frères Editions, p. 11-25.

9. NAWATHE D.R., LAMORDE A.G., 1985. Rinderpest in Nigeria: the unfinished story. Vet. Rec., 117: 669.

10. NGANGNOU A., ZOYEM N., 1994. Evaluation de la protection vaccinale contre la peste bovine au Cameroun. I. La Province de I'Adamaoua. Revue Elev. M éd. vét. Pays trop., 47 : 35-37.

11. PLOWRIGHT W., FERRIS R.D., 1961. Studies with rinderpest virus in tissue culture. III. The stability of cultured virus and its use in viral neutralisation tests. Arch. ges. Virusforch., 11: 516-533.

12. ROSSITER P.B., JESSET D.M., 1982. Microtitre techniques for the assay of rinderpest virus neutralizing antibody. Res. Vet. Sci., 32: 253-256.

13. ROSSITER P.B., JESSET D.M., WAFU LA J.S., KARSTAD L., CHEMA S., TAYLOR W.P., ROWE L., NYANGE J.C., OTARU M., MUMBALA M., SCOTT G.R., 1983. Reemergence of rinderpest as a threat in East Africa since 1979. Vet. Rec., 113: 459-461.

14. ROSSITER P.B., WAMWAYI H.M., 1989. Surveillance and monitoring programmes for the control of rinderpest: a review. Trop. Anim. Health Prod., 21: 89-99.

15. SCOTT G.R., 1981. Rinderpest and peste des petits ruminants. In: Gibbs E.P.J. ed., Virus diseases of food animals. London, UK, Academic Press, p. 401-432.

16. STRICKLAND K.L., 1962. Vaccination of calves against rinderpest. Vet. Rec., 74: 630-631.

17. YILMA T., HSU D., JONES L., OWENS S., GRUGMAN M., MEBUSC., YAMANAKA M., DALE B., 1988. Protection of cattle against rinderpest with vaccinia virus recombinants expressing the $H A$ or $F$ gene. Science, 242: 1058-1061.

Reçu le 30.9.97, accepté le 25.5.98 


\section{Summary}

Tanya V.N., Keng A., Messine O., Ngangnou A., Zoyem N., Kulu I.N.D. Dynamics of passive immunity and strategy of vaccination of calves against rinderpest

To determine the rate of decay of maternally derived rinderpest virus antibodies, calves born of immune dams were serologically monitored from birth until one year of age. The potential interference of these antibodies with vaccination was evaluated by vaccinating calves possessing a wide range of maternal antibodies. The maternal antibodies diminished progressively with age. At about 8 months of age, only $7 \%$ of the calves still had maternal antibodies. After this age, maternal antibodies were not detectable. Regression analysis of the maternal antibody titer with age indicated a half life of 48.01 days. Maternally derived antibodies depressed the response to vaccination. This response was inversely proportional to the level of maternal antibodies present at the time of vaccination. It was also observed that calves with very high maternal antibody titers did not respond to vaccination and their maternal antibody titers even decreased. These results are important in the planning of rinderpest eradication programs.

Key words: Cattle - Calf - Rinderpest - Vaccination - Passive immunity - Immunology - Neutralization - Antibody Cameroon.

\section{Resumen}

Tanya V.N., Keng A., Messine 0., Ngangnou A., Zoyem N., Kulu I.N.D. Dinámica de la inmunidad pasiva y estrategia de vacunación de terneros contra la peste bovina

Con el fin de determinar la dinámica de la inmunidad pasiva contra la peste bovina, se siguieron serológicamente teneros nacidos de madres inmunizadas, desde el nacimiento hasta un año de edad. Se evaluó la eventual interferencia de estos anticuerpos con la vacunación, mediante una vacunación de los terneros que presentaban un gran abanico de títulos de anticuerpos. Los anticuerpos de origen materno disminuyeron progresivamente con la edad. A 8 meses, solo $7 \%$ de los terneros los poseían aún. Después de esta edad, estos anticuerpos no fueron detectables. El análisis por regresión del título de anticuerpos maternos con la edad de los teneros, mostró una vida media de 48,01 días. Estos anticuerpos deprimieron la respuesta a la vacunación, la cual fue inversamente proporcional al nivel de anticuerpos maternos presentes en el momento de la vacunación. Se observó igualmente que los terneros con un título de anticuerpos maternos elevados, no solamente no respondieron a la vacunación, sino que el título serológico también disminuyó. Estos resultados son importantes para el planeamiento de campañas de erradicación de la peste bovina.

Palabras clave: Ganado bovino - Ternero - Peste bovina Vacunación - Inmunidad pasiva - Inmunología Neutralización - Anticuerpo - Camerún. 